\title{
Azimuthal asymmetries in single polarized proton-proton Drell-Yan processes
}

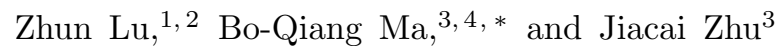 \\ ${ }^{1}$ Department of Physics, Southeast University, Nanjing 211189, China \\ ${ }^{2}$ Departamento de Física, Universidad Técnica Federico Santa María, and \\ Centro Científico-Tecnológico de Valparaíso Casilla 110-V, Valparaíso, Chile \\ ${ }^{3}$ School of Physics and State Key Laboratory of Nuclear Physics and Technology, \\ Peking University, Beijing 100871, China \\ ${ }^{4}$ Center for High Energy Physics, Peking University, Beijing 100871, China
}

\begin{abstract}
We study the azimuthal asymmetries in proton-proton Drell-Yan processes with one incident proton being transversely or longitudinally polarized. We consider particularly the asymmetries contributed by the leading-twist chiral-odd quark distributions. We analyze the asymmetries with $\sin \left(2 \phi+\phi_{S}\right)$ and $\sin \left(2 \phi-\phi_{S}\right)$ modulations in transverse single polarized $p^{\uparrow} p$ Drell-Yan and $\sin 2 \phi$ asymmetries in longitudinal single polarized $p^{\rightarrow} p$ Drell-Yan at the Relativistic Heavy Ion Collider, the Japan Proton Accelerator Research Complex, E906 (Fermi Lab), and the Nuclotron-based Ion Collider Facility (Joint Institute for Nuclear Research). We show that the measurements of the asymmetries in those facilities can provide valuable information of the chiral-odd structure of the nucleon both in the valence and sea regions.
\end{abstract}

PACS numbers: 12.39.Ki, 13.85.Qk, 13.88.+e, 13.85.-t

\section{INTRODUCTION}

The single spin asymmetry (SSA) appearing in various high-energy scattering processes [1-4] is among the most challenging issues of QCD spin physics. Large SSAs were observed experimentally in the process $p p^{\uparrow} \rightarrow \pi X$ [5] two decades ago. Standard perturbative QCD based on collinear factorization to leading power of $1 / Q$ cannot explain these asymmetries [6]. Many theoretical studies [7-9] have been proposed to explain the origin of such asymmetries. One standard approach is to assume the existence of parton distribution and/or fragmentation depending on intrinsic transverse momentum, by going beyond the collinear picture. In this transverse momentum dependent (TMD) framework, novel structures of the nucleon emerge. For instance, due to the correlation of nucleon transverse spin $\boldsymbol{S}$ and quark transverse momentum $\boldsymbol{k}_{T}$, there can be an asymmetric distribution of unpolarized quarks in a transversely polarized proton [10]:

$$
\begin{aligned}
& f_{q / p^{\uparrow}}\left(x, k_{T}\right)-f_{q / p^{\uparrow}}\left(x,-k_{T}\right) \\
= & \Delta^{N} f_{q / p^{\uparrow}}\left(x, k_{T}^{2}\right) \frac{\left(\hat{\boldsymbol{P}} \times \boldsymbol{k}_{T}\right) \cdot \boldsymbol{S}}{\left|\boldsymbol{k}_{T}\right|} \\
= & -2 f_{1 T}^{\perp q}\left(x, k_{T}^{2}\right) \frac{\left(\hat{\boldsymbol{P}} \times \boldsymbol{k}_{T}\right) \cdot \boldsymbol{S}}{M} .
\end{aligned}
$$

Here $f_{1 T}^{\perp q}$ or $\Delta^{N} f_{q / p^{\uparrow}}$ is referred to as the Sivers function [8, 9], and has been applied to explain the SSAs observed in the process $p p^{\uparrow} \rightarrow \pi X$. For a while the Sivers function was thought to be forbidden by the time-reversal invariance property of QCD [11]. However, model calculations 12 by Brodsky, Hwang and Schmidt show that

*Electronic address: mabq@pku.edu.cn the Sivers effect can be allowed in the semi-inclusive deeply inelastic scattering (SIDIS) and Dell-Yan process at leading-twist level, due to the final/initial state interaction (FSI/ISI) between the struck quark and the target remnant. It was then realized that FSI/ISI can be accumulated into the Wilson lines (gauge-links) that are the key ingredients for a full gauge-invariant definition [13, 14] of TMD distribution functions. This also leads to the prediction on the sign reversal of the Sivers functions in SIDIS and Drell-Yan [15]. For hadron productions in hadron-hadron collision (i.e., $H_{A}+H_{B} \rightarrow$ $\left.h_{1}+h_{2}+X\right)$, the situation is more involved, as there are colored objects in both the initial state and the final state. The multiple FSI/ISI will generate processdependent TMD distributions 16-19] which are different from those in SIDIS or Drell-Yan process. This is also viewed as the breakdown of the generalized TMD factorization in inclusive hadro-production of hadrons [20].

Allowing naive- $T$-odd parton distributions encourages a lot of theoretical and experimental studies. Substantial SSAs contributed by the Sivers effect in SIDIS processes 21 28], with one colliding nucleon transversely polarized, have been measured by several experiments during recent years. The asymmetries are identified by the angular dependence $\sin \left(\phi_{h}-\phi_{S}\right)$, where $\phi_{h}$ and $\phi_{S}$ denote, respectively, the azimuthal angles of the produced hadron and of the nucleon spin polarization, with respect to the lepton scattering plane. The data on the Sivers SSAs have been utilized by different groups 29 33] to extract the Sivers functions of the proton, on the basis of the TMD factorization [34, 35]. Those sets of parametrizations of the Sivers functions were applied to predict the Sivers SSA in various processes, such as the SIDIS at Jefferson Lab (JLab), and the Drell-Yan processes at the COmmon Muon Proton Apparatus for Structure and Spectroscopy, the Relativistic Heavy Ion Collider (RHIC), and the Polarized Antiproton eXper- 
iment (PAX). Many planned measurements of SSAs in single polarized Drell-Yan processes at the established or planned hadron accelerators/colliders have been proposed. One of the main goals of these experiments is to test the sign change of the Sivers functions in SIDIS and Drell-Yan process [30, 36], as a crucial prediction of QCD dynamics. It is also worthwhile to mention that a sign mismatch for the $k_{T}$-moments of Sivers functions has been found when the authors of Ref. [37] compared the functions extracted from SIDIS data and those extracted from $p^{\uparrow} p \rightarrow \pi X$ data.

The planned polarized Drell-Yan processes at (future) available facilities also provide great opportunities to investigate various spin and transverse momentum dependent (TMD) distributions. Besides the Sivers effect, there are some other effects that may contribute to the azimuthal spin asymmetries at leading twist thereby could be measured in single polarized Drell-Yan processes. It is interesting to point out that all these leadingtwist effects (except the Sivers effect) involve the chiralodd parton distribution functions. For example, the following combinations

$$
h_{1} \otimes h_{1}^{\perp}, \quad h_{1 T}^{\perp} \otimes h_{1}^{\perp}, \quad h_{1 L}^{\perp} \otimes h_{1}^{\perp},
$$

will lead to SSAs with $\sin \left(2 \phi-\phi_{S}\right), \sin \left(2 \phi+\phi_{S}\right)$ and $\sin 2 \phi$ angular dependences, respectively. Here $\phi$ and $\phi_{S}$ are the azimuthal angles of the dilepton pair and proton transverse spin with respect to the hadron plane, and we use the convention for the angle definition introduced in Ref. [38]. These types of the asymmetries arise from the coupling of two different chiral-odd parton distributions. The coupling $h_{1} \otimes h_{1}^{\perp}$ was first introduced and analyzed in Ref. [39] as an alternative mechanism for SSA and a method of accessing the transversity distribution functions $h_{1}$ [40, 41]. The key ingredient for these SSAs is the Boer-Mulders function $h_{1}^{\perp}$ [42], which is also a naive- $T$-odd TMD distribution function and provides the necessary phase required for SSA. In this paper, we will present a phenomenological analysis of these SSAs in the proton-proton Drell-Yan process contributed by various leading-twist chiral-odd distribution functions. We consider proton-proton induced polarized Drell-Yan process, since there are several hadron accelerators/colliders, such as RHIC, the Japan Proton Accelerator Research Complex (J-PARC), E906 at Fermi Lab, and the Nuclotronbased Ion Collider fAcility (NICA) at the Joint Institute for Nuclear Research (JINR), that can perform these experiments. Therefore the asymmetries at different energies and kinematical regions can be analyzed and compared, which is important for obtaining the information of various chiral-odd distributions functions from experiments.

The remaining content of the paper is organized as follows. In Sec. II, we briefly review the systematics of leading-twist chiral-odd TMD quark distributions, then give the expressions of the corresponding azimuthal angle weighted asymmetries $A_{T U}^{\sin \left(2 \phi-\phi_{S}\right)}, A_{T U}^{\sin \left(2 \phi+\phi_{S}\right)}$ and $A_{L U}^{\sin 2 \phi}$ in the framework of TMD factorization. We consider both the single longitudinally and transversely polarized Drell-Yan processes. In Sec. III, we present the phenomenological predictions for single transverse spin asymmetry in $p^{\uparrow} p$ Drell-Yan process, and single longitudinal spin asymmetry in $p \rightarrow p$ Drell-Yan process at RHIC, J-PARC, E906 and NICA. We conclude our paper in Sec. IV.

\section{SYSTEMATICS OF LEADING-TWIST CHIRAL-ODD DISTRIBUTIONS AND THEIR ROLES IN SSAS}

At leading twist, according to the hermiticity properties of the fields and parity invariance, one may decompose the TMD quark-quark correlation matrix of the nucleon as follows 42 45]

$$
\begin{aligned}
\Phi\left(x, \boldsymbol{k}_{T}\right)=\frac{1}{2}\{ & f_{1} \not h_{+}-f_{1 T}^{\perp} \frac{\epsilon_{T}^{\rho \sigma} k_{T \rho} S_{T \sigma}}{M} \not h_{+}+\left(S_{L} g_{1 L}-\frac{k_{T} \cdot S_{T}}{M} g_{1 T}\right) \gamma_{5} \not \not_{+} \\
& \left.+h_{1 T} \frac{\left[\phi_{T}, \not h_{+}\right] \gamma_{5}}{2}+\left(S_{L} h_{1 L}^{\perp}-\frac{k_{T} \cdot S_{T}}{M} h_{1 T}^{\perp}\right) \frac{\left[\not k_{T}, \not h_{+}\right] \gamma_{5}}{2 M}+i h_{1}^{\perp} \frac{\left[\not k_{T}, \not h_{+}\right]}{2 M}\right\} .
\end{aligned}
$$

Here $n_{+}=\left(0,1, \mathbf{0}_{T}\right)$ is a lightlike vector expressed in the light-cone coordinates, in which an arbitrary four-vector $a$ is written as $\left\{a^{-}, a^{+}, \boldsymbol{a}_{T}\right\}$, with $a^{ \pm}=\left(a^{0} \pm a^{3}\right) / \sqrt{2}$ and $\boldsymbol{a}_{T}=\left(a^{1}, a^{2}\right)$. The eight functions on the right-hand side of Eq. (3) not only depend on longitudinal momentum fraction $x$, but also on the intrinsic transverse momentum of the quark $\boldsymbol{k}_{T}$. Therefore they are named as transverse momentum dependent (TMD) distributions, or alternatively the three-dimensional parton distribution functions (3dPDFs) in momentum space. As the extensions of the usual Feynman distribution functions, $3 \mathrm{dPDF}$ enter the description of various semi-inclusive reactions and encode a wealth of new information on the nucleon structures that cannot be described merely by the leading-twist collinear picture. 

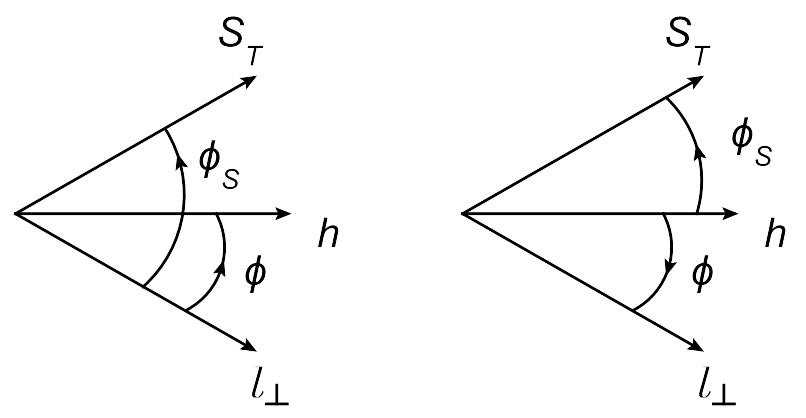

FIG. 1: Left panel: definition of azimuthal angles in Refs. [39] and 63]; right panel: definition of azimuthal angles in Ref. [38]. Using the replacements $\phi \rightarrow-\phi$ and $\phi_{S} \rightarrow \phi_{S}-\phi$, the definition in the left panel is transformed to that in the right panel.

Each of these eight 3dPDFs represents a special parton structure of the nucleon. Five of them, the Sivers function $f_{1 T}^{\perp}$, the Boer-Mulders function $h_{1}^{\perp}$, the pretzelosity $h_{1 T}^{\perp}$, the transversal helicity $g_{1 T}$, and the longitudinal transversity $h_{1 L}^{\perp}$, vanish upon integrating $\Phi\left(x, \boldsymbol{k}_{T}\right)$ over $\boldsymbol{k}_{T}$. Particularly, two $3 \mathrm{dPDF}$, the Sivers function and the Boer-Mulders function are naive- $T$-odd distributions and account for the SSAs in various processes. Among the eight $3 \mathrm{dPDFs}, h_{1 T}, h_{1}^{\perp}, h_{1 T}^{\perp}$, and $h_{1 L}^{\perp}$ are chirally odd, that is, they describe densities of the probed quarks with helicity flipped. Except $h_{1}^{\perp}$, other three chiral-odd distribution are $T$-even. The relation between $h_{1 T}$ given in Eq. (3) and $h_{1}$ is

$$
h_{1}\left(x, k_{T}^{2}\right)=h_{1 T}\left(x, k_{T}^{2}\right)+\frac{\boldsymbol{k}_{T}^{2}}{2 M^{2}} h_{1 T}^{\perp}\left(x, k_{T}^{2}\right) .
$$

Since $h_{1}$ naturally appears in the expression of related azimuthal asymmetries, our discussion on the transversity in the rest of our paper is based on $h_{1}$ rather than $h_{1 T}$. The distributions $h_{1}$ and $h_{1}^{\perp}$ describe the densities of transversely polarized quarks inside a transversely polarized proton and an unpolarized proton, respectively. The distributions $h_{1 T}^{\perp}$ and $h_{1 L}^{\perp}$ arise from double spin correlations in the parton distribution functions (PDFs), representing the densities of transversely polarized quarks in a transversely (but in a different direction) polarized proton and a longitudinally polarized proton, respectively.

Because of the chiral-odd nature of $h_{1}, h_{1}^{\perp}, h_{1 T}^{\perp}$ and $h_{1 L}^{\perp}$, in high-energy processes they have to combine together with another chiral-odd object, i.e., with the Collins fragmentation function in SIDIS, or with another chiral-odd distribution function in Drell-Yan, to manifest their effects. This makes them rather difficult to be probed experimentally. As a result, they are less known than the chiral-even distribution functions. Anyway, there are some efforts to extract transversity from SIDIS data [46, 47], and Boer-Mulders function from SIDIS and Drell-Yan data [48 51]. For $h_{1 T}^{\perp}$ and $h_{1 L}^{\perp}$, there are extensive model calculations [52 60 ] and some proposals to measure them in SIDIS and $p \bar{p}$ Drell-Yan processes.

All the leading-twist chiral-odd parton distributions can be probed in single polarized proton-proton DrellYan processes:

$$
p^{\uparrow / \rightarrow}\left(P_{1}\right)+p\left(P_{2}\right) \rightarrow \gamma^{*}(q)+X \rightarrow \ell(l)+\bar{\ell}\left(l^{\prime}\right)+X .
$$

Here we assume that one proton (with momentum $P_{1}$ ) is polarized, and $\uparrow$ or $\rightarrow$ denotes its transverse polarization or longitudinal polarization. In leading order, the dilepton pair is produced from the annihilation of the quark and antiquark from each proton. We denote the momenta of the annihilating partons from polarized proton and unpolarized proton as $k_{1}$ and $k_{2}$, respectively. Then we can define the kinematical variables as

$$
\begin{aligned}
q & =l+l^{\prime}=\left(q^{0}, \boldsymbol{q}_{T}, q^{3}\right), \quad Q^{2}=q^{2}, \\
x_{1} & =\frac{Q^{2}}{2 P_{1} \cdot q} \approx \frac{k_{1}^{+}}{P_{1}^{+}}, \quad x_{1}=\frac{Q^{2}}{2 P_{2} \cdot q} \approx \frac{k_{2}^{-}}{P_{2}^{-}}, \\
y & =\frac{1}{2} \ln \left(\frac{x_{1}}{x_{2}}\right) .
\end{aligned}
$$

In the Drell-Yan process, if the transverse momentum of the dilepton $\boldsymbol{q}_{T}$ is measured, we can apply the TMD factorization [34, 35, 61] which is valid in the region $q_{T}^{2} \ll Q^{2}$ to write down the differential cross section of processes at leading order as [38, 39]

$$
\begin{aligned}
\frac{d \sigma}{d x_{1} d x_{2} d^{2} \boldsymbol{q}_{T} d \Omega}= & \frac{\alpha_{e m}^{2}}{3 Q^{2}}\left\{\frac{\left(1+\cos ^{2} \theta\right)}{4} F_{U U}^{1}+S_{L} \frac{\sin ^{2} \theta}{4} \sin 2 \phi F_{L U}^{\sin 2 \phi}\right. \\
& \left.+\left|\boldsymbol{S}_{T}\right| \frac{\sin ^{2} \theta}{4}\left[\sin \left(2 \phi+\phi_{S}\right) F_{T U}^{\sin \left(2 \phi+\phi_{S}\right)}+\sin \left(2 \phi-\phi_{S}\right) F_{T U}^{\sin \left(2 \phi-\phi_{S}\right)}\right]+\cdots\right\}
\end{aligned}
$$

Here $\phi$ and $\phi_{S}$ are the azimuthal angles of $\boldsymbol{l}_{\perp}$ and $\boldsymbol{S}_{T}$ with respect to the normalized vector $\boldsymbol{h}=\boldsymbol{q}_{T} / Q_{T}$, respectively; and $d \Omega=d \cos \theta d \phi$ is the solid angle of the lepton $\ell$ in the center-of-mass system of the lepton pair. In Eq. (7) we only give the terms appearing in (5), and other terms do not contribute in our analysis below. We 

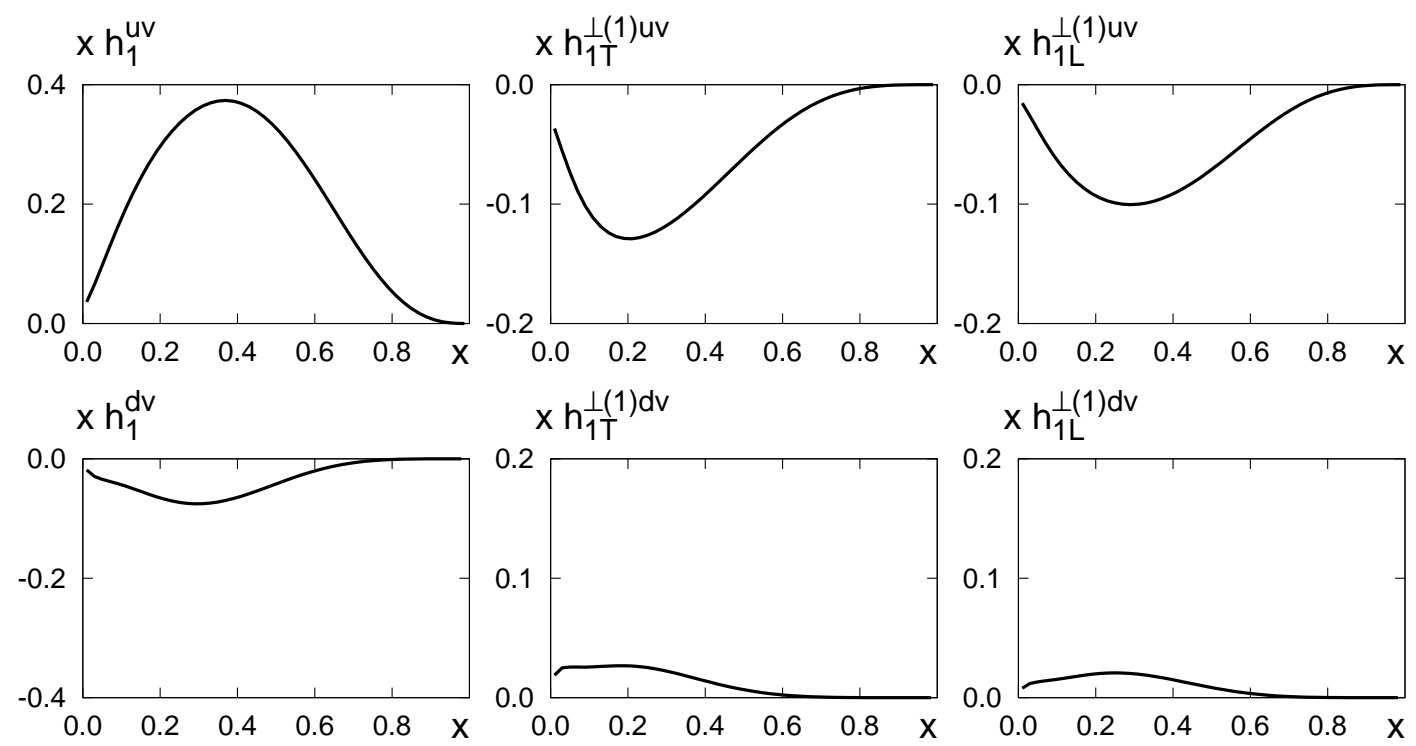

FIG. 2: The light-cone diquark model results of $x h_{1}(x)$ (left panels), $x h_{1 T}^{\perp(1)}(x)$ (central panels), and $x h_{1 T}^{\perp(1)}(x)$ (right panels) of valence $u$ and $d$ quarks at $Q^{2}=1 \mathrm{GeV}^{2}$, respectively.

note that in the literature there are different definitions of angles $\phi$ and $\phi_{S}$, as shown in Fig. 1. In this work we adopt the definition in Ref. [38]. Also, we apply the so-called Collins-Soper frame [62], in which the structure functions are expressed as

$$
\begin{aligned}
F_{U U}^{1} & =\mathcal{C}\left[f_{1} \bar{f}_{1}\right], \\
F_{T U}^{\sin \left(2 \phi-\phi_{S}\right)} & =\mathcal{C}\left[\frac{\boldsymbol{h} \cdot \boldsymbol{k}_{1 T}}{M_{N}} h_{1} \bar{h}_{1}^{\perp}\right], \\
F_{T U}^{\sin \left(2 \phi+\phi_{S}\right)} & =\mathcal{C}\left[\frac{2\left(\boldsymbol{h} \cdot \boldsymbol{k}_{1 T}\right)\left[2\left(\boldsymbol{h} \cdot \boldsymbol{k}_{1 T}\right)\left(\boldsymbol{h} \cdot \boldsymbol{k}_{2 T}\right)-\boldsymbol{k}_{1 T} \cdot \boldsymbol{k}_{2 T}\right]-k_{1 T}^{2}\left(\boldsymbol{h} \cdot \boldsymbol{k}_{2 T}\right)}{2 M_{N}^{3}} h_{1 T}^{\perp} \bar{h}_{1}^{\perp}\right], \\
F_{L U}^{\sin 2 \phi} & =\mathcal{C}\left[\frac{2\left(\boldsymbol{h} \cdot \boldsymbol{k}_{1 T}\right)\left(\boldsymbol{h} \cdot \boldsymbol{k}_{2 T}\right)-\boldsymbol{k}_{1 T} \cdot \boldsymbol{k}_{2 T}}{M_{N}^{2}} h_{1 L}^{\perp} \bar{h}_{1}^{\perp}\right] .
\end{aligned}
$$

In above equations we have used the notation

$$
\begin{aligned}
\mathcal{C}\left[w\left(\boldsymbol{k}_{1 T}, \boldsymbol{k}_{2 T}\right) f \bar{g}\right]= & \sum_{q} e_{q}^{2} \int d^{2} \boldsymbol{k}_{1 T} d^{2} \boldsymbol{k}_{2 T} \\
& \times \delta^{(2)}\left(\boldsymbol{q}_{T}-\boldsymbol{k}_{1 T}-\boldsymbol{k}_{2 T}\right) w\left(\boldsymbol{k}_{1 T}, \boldsymbol{k}_{2 T}\right) \\
& \times\left[f^{q}\left(x_{1}, k_{1 T}^{2}\right) g^{\bar{q}}\left(x_{2}, k_{2 T}^{2}\right)\right. \\
& \left.+f^{\bar{q}}\left(x_{1}, k_{1 T}^{2}\right) g^{q}\left(x_{2}, k_{2 T}^{2}\right)\right] .
\end{aligned}
$$

Thus all structure functions depend on $x_{1}, x_{2}$ and $q_{T}=$ $\left|\boldsymbol{q}_{T}\right|$.

As shown in Eq. (7), the structure functions $F_{T U}^{\sin \left(2 \phi-\phi_{S}\right)}$ and $F_{T U}^{\sin \left(2 \phi+\phi_{S}\right)}$ contribute to the cross section in the case in which one proton is transversely polarized (denoted by subscript $T$ ), and will give rise to $\sin \left(2 \phi-\phi_{S}\right)$ and $\sin \left(2 \phi+\phi_{S}\right)$ angular dependences, respectively. The structure function $F_{L U}^{\sin 2 \phi}$ contribute to the cross section in the case in which one proton is longitudinally polarized (denoted by the subscript $L$ ), and will give rise to a $\sin 2 \phi$ angular dependence. Therefore one can define the following azimuthal asymmetries

$$
\begin{aligned}
A_{T U}^{\sin \left(2 \phi-\phi_{S}\right)}\left(x_{1}, x_{2}, q_{T}\right) & =\frac{F_{T U}^{\sin \left(2 \phi-\phi_{S}\right)}}{F_{U U}^{1}}, \\
A_{T U}^{\sin \left(2 \phi+\phi_{S}\right)}\left(x_{1}, x_{2}, q_{T}\right) & =\frac{F_{T U}^{\sin \left(2 \phi+\phi_{S}\right)}}{F_{U U}^{1}}, \\
A_{L U}^{\sin 2 \phi}\left(x_{1}, x_{2}, q_{T}\right) & =\frac{F_{L U}^{\sin 2 \phi}}{F_{U U}^{1}} .
\end{aligned}
$$


Our definitions for the azimuthal asymmetries are similar to the analyzing power given in [39] and are different from the transverse momentum weighted asymmetries defined in 63]. For experimental measurement of the asymmetries given in Eqs.(13), (14), and (15), the polar angle $\theta$ of the lepton $\ell$ should be identified. As a compensation, larger asymmetries could be measured.

One can also express the cross section of the Drell-Yan process, depending on $y$ and $Q^{2}$ as

$$
\frac{d \sigma}{d y d Q^{2} d^{2} \boldsymbol{q}_{T} d \Omega}=\frac{1}{s} \frac{d \sigma}{d x_{1} d x_{2} d^{2} \boldsymbol{q}_{T} d \Omega} .
$$

At the region $\boldsymbol{q}_{T}^{2} \ll Q^{2}$, the following relations hold

$$
x_{1}=\frac{Q}{\sqrt{s}} e^{y}, \quad x_{2}=\frac{Q}{\sqrt{s}} e^{-y} .
$$

Therefore we can define the $y$-dependent and $Q^{2}$ dependent SSAs as

$$
\begin{aligned}
A_{P U}^{a}(y) & =\frac{\int d^{2} \boldsymbol{q}_{T} d Q^{2} \frac{1}{Q^{2}} F_{P U}^{a}}{\int d^{2} \boldsymbol{q}_{T} d Q^{2} \frac{1}{Q^{2}} F_{U U}^{1}}, \\
A_{P U}^{a}\left(Q^{2}\right) & =\frac{\int d^{2} \boldsymbol{q}_{T} d y F_{P U}^{a}}{\int d^{2} \boldsymbol{q}_{T} d y F_{U U}^{1}},
\end{aligned}
$$

where we have used the short notes $P=T$ or $L$, and $a=$ $\sin \left(2 \phi \pm \phi_{S}\right)$ for $P=T$ and $a=\sin 2 \phi$ for $P=L$. The integrations in Eqs. (18) and (19) are performed according to kinematical cuts or experimental acceptances.

\section{PHENOMENOLOGICAL ANALYSIS OF AZIMUTHAL ASYMMETRIES AT RHIC, J-PARC, E906 AND NICA}

In this section we investigate the prospects of experimental measurements on the azimuthal asymmetries defined in the last section at various facilities that can conduct single polarized proton-proton Drell-Yan processes. The proton-proton Drell-Yan process involves the annihilation of a quark from one proton and a antiquark from another proton. In order to calculate $F_{P U}^{a}$, one needs to know the distributions $h_{1}, h_{1 T}^{\perp}, h_{1 L}^{\perp}$, and $h_{1}^{\perp}$ of both the valence and sea quarks. Although there are some extractions of transversity and Boer-Mulders functions from SIDIS and Drell-Yan data, most of the chiral-odd parton distributions are not measured and less known, especially those of sea quarks. In order to estimate the azimuthal asymmetries in $p p$ Drell-Yan processes, we apply the following ansatz:

- For the Boer-Mulders functions $h_{1}^{\perp q}\left(x, k_{T}^{2}\right)$, we adopt the result extracted from the unpolarized $p d$ [64] and $p p$ 65] Drell-Yan data in Ref. [49], as there is parameterization for both valence and sea quarks with the following form:

$$
\begin{aligned}
h_{1}^{\perp q}\left(x, k_{T}^{2}\right)= & H_{q} x^{c^{q}}(1-x)^{b} f_{1}^{q}(x) \\
& \times \frac{1}{\pi k_{b m}^{2}} \exp \left(\frac{-k_{T}^{2}}{k_{b m}^{2}}\right),
\end{aligned}
$$

where the subscript "bm" stands for the BoerMulders functions, and $q=u, d, \bar{u}$, and $\bar{d}$. We have ignored the contributions from other flavors, since they are assumed to be small. We note that the possible range of parameters $H_{q}$ allowed by the positivity bound for $h_{1}^{\perp}$ can be described by the coefficient $\omega$, namely, that the substitutions $H_{q} \rightarrow \omega H_{q}$ for $q=u, d$ and $H_{q} \rightarrow \frac{1}{\omega} H_{q}$ for $q=\bar{u}, \bar{d}$ will not change the calculated $\cos 2 \phi$ asymmetry (contributed by $h_{1}^{\perp q} \times h_{1}^{\perp \bar{q}}$ ) in the unpolarized $p d$ and $p p$ Drell-Yan data. The range of $\omega$ given in Ref. 49] is $0.48<\omega<2.1$ and $\omega=1$ corresponds to the central values of $H_{q}$. However, for the azimuthal asymmetries given in Eqs. (18) and (19), the variation of $\omega$ will lead to the change of the magnitudes of the asymmetries, and will be considered in our calculations.

- For the $T$-even distributions $h_{1}, h_{1 T}^{\perp}$, and $h_{1 L}^{\perp}$ of valence quarks, there are considerable model calculations. We will deploy the calculation from the light-cone quark-diquark model. In this model, the Melosh-Wigner rotation [66], which plays an important role to understand the proton spin puzzle 67] due to the relativistic effect of quark transversal motions, has been taken into account. In practice, the light-cone quark-diquark model has been applied to calculate the helicity distributions [68], the transversity distributions [69, 70] and other 3dPDFs [55, 59, 71], and related azimuthal spin asymmetries in SIDIS processes [72, 73].

The light-cone model results for the distributions $h_{1}^{q v}\left(x, k_{T}^{2}\right), h_{1 T}^{\perp q v}\left(x, k_{T}^{2}\right)$, and $h_{1 L}^{\perp q v}\left(x, k_{T}^{2}\right)$ are given as [55, 59, 69, 70]

$$
\begin{aligned}
j^{u v}\left(x, k_{T}^{2}\right)= & {\left[f_{1}^{u v}\left(x, k_{T}^{2}\right)-\frac{1}{2} f_{1}^{d v}\left(x, k_{T}^{2}\right)\right] W_{S}^{j}\left(x, k_{T}^{2}\right) } \\
& -\frac{1}{6} f_{1}^{d v}\left(x, k_{T}^{2}\right) W_{V}^{j}\left(x, k_{T}^{2}\right) \\
j^{d v}\left(x, k_{T}^{2}\right)= & -\frac{1}{3} f_{1}^{d v}\left(x, k_{T}^{2}\right) W_{V}^{j}\left(x, k_{T}^{2}\right)
\end{aligned}
$$

where $j=h_{1}, h_{1 T}^{\perp}, h_{1 L}^{\perp}$, respectively, and the superscript " $v$ " is corresponding to the valence distributions. $W_{S / V}^{j}\left(x, k_{T}^{2}\right)$ are the rotation factors for the scalar or axial vector spectator-diquark cases. Their explicit form are

$$
\begin{array}{r}
W_{D}^{h_{1}}\left(x, k_{T}^{2}\right)=\frac{\left(x \mathcal{M}_{D}+m_{q}\right)^{2}}{\left(x \mathcal{M}_{D}+m_{q}\right)^{2}+k_{T}^{2}}, \\
W_{D}^{h_{1 T}^{\perp}}\left(x, k_{T}^{2}\right)=-\frac{2 M_{N}^{2}}{\left(x \mathcal{M}_{D}+m_{q}\right)^{2}+k_{T}^{2}}, \\
W_{D}^{h_{1 L}^{\perp}}\left(x, k_{T}^{2}\right)=-\frac{2 M_{N}\left(x \mathcal{M}_{D}+m_{q}\right)}{\left(x \mathcal{M}_{D}+m_{q}\right)^{2}+k_{T}^{2}},
\end{array}
$$

with

$$
\mathcal{M}_{D}=\sqrt{\frac{m_{q}^{2}+k_{T}^{2}}{x}+\frac{m_{D}^{2}+k_{T}^{2}}{1-x}} .
$$



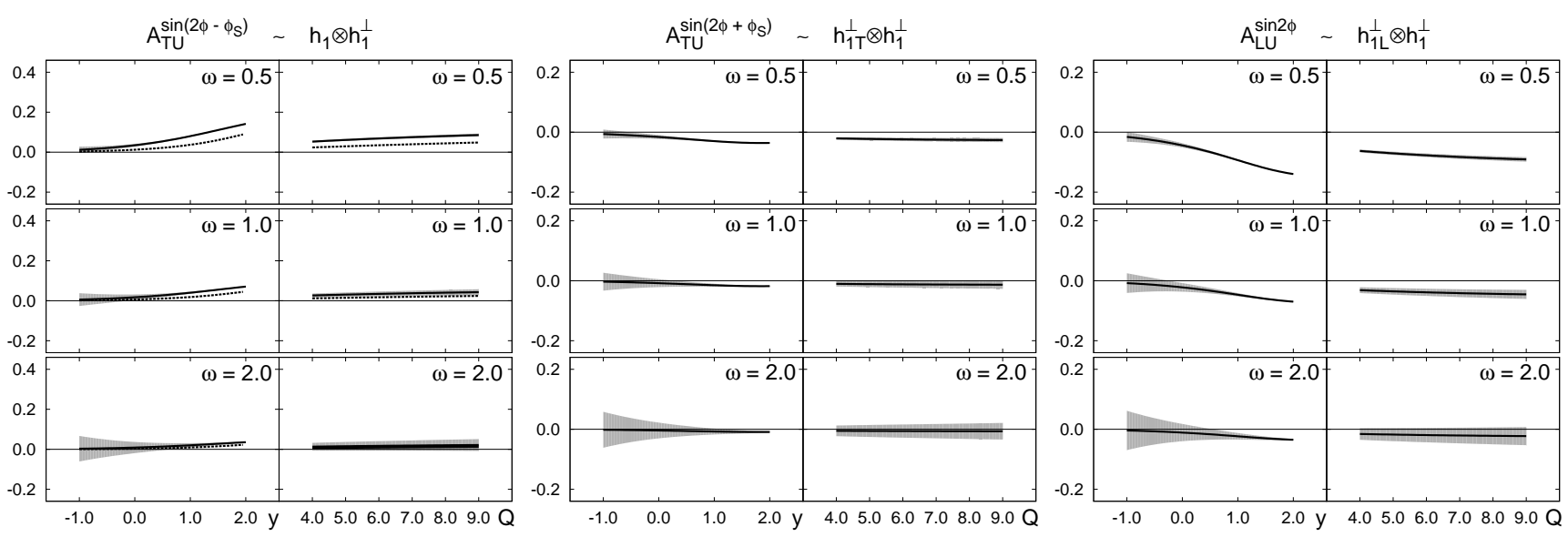

FIG. 3: Azimuthal asymmetries $A_{T U}^{\sin \left(2 \phi-\phi_{S}\right)}$ (left panels), $A_{T U}^{\sin \left(2 \phi+\phi_{S}\right)}$ (central panels), and $A_{T U}^{\sin 2 \phi}$ (right panels) at RHIC collider experiments as functions of the rapidity $y$ and the dilepton mass $Q$, respectively. The dashed lines in the left panels correspond to the contributions from the valence transversity distributions $h_{1}$ fitted by Anselmino et al. in Ref. [47]. The thick solid lines in the left, central, and right panels represent the contributions from the distributions $h_{1}, h_{1 T}^{\perp}$, and $h_{1 L}^{\perp}$ of valence quarks alone in the light-cone quark-diquark model [55, [59, 69, 70]. The shaded regions give the ranges of the asymmetries by considering the additional contribution from the distributions $h_{1}, h_{1 T}^{\perp}$, and $h_{1 L}^{\perp}$ of sea quarks constrained by the positivity bounds given in Eqs. (27), (28) and (29). The upper and lower limits of the bands correspond to the asymmetries by saturating the positivity bounds.

An important feature manifested by these rotation factors is that they automatically satisfy the requirement of the positivity bounds [74] for the PDFs. In the left, central, and right panels of Fig. 2 we plot the curves for $x h_{1}(x), x h_{1 T}^{\perp(1)}(x)$, and $x h_{1 T}^{\perp(1)}(x)$ of valence $u$ and $d$ quarks at $Q^{2}=1$ $\mathrm{GeV}^{2}$, respectively.

As there is already extraction of transversity from the global analysis by combining the SIDIS and $e^{+} e^{-}$annihilation data, we also use the most recent parametrizations [47] for $h_{1}$ to calculate the asymmetries $A_{T U}^{\sin 2 \phi}$, and compare the results with those predicted from our model calculation.

- In order to consider the effects of the distributions $h_{1}, h_{1 T}^{\perp}$ and $h_{1 L}^{\perp}$ of sea quarks, we constrain them by the positivity bounds 74 ]

$$
\begin{aligned}
\left|h_{1}^{\bar{q}}\left(x, k_{T}^{2}\right)\right| & \leqslant f_{1}^{\bar{q}}\left(x, k_{T}^{2}\right), \\
\left|\frac{k_{T}^{2}}{2 M_{N}^{2}} h_{1 T}^{\perp \bar{q}}\left(x, k_{T}^{2}\right)\right| & \leqslant f_{1}^{\bar{q}}\left(x, k_{T}^{2}\right), \\
\left|\frac{k_{T}}{M_{N}} h_{1 L}^{\perp \bar{q}}\left(x, k_{T}^{2}\right)\right| & \leqslant f_{1}^{\bar{q}}\left(x, k_{T}^{2}\right) .
\end{aligned}
$$

They give rise to additional contributions to the asymmetries through the coupling $j^{\bar{q}} \otimes h_{1}^{\perp q}$, and will give a range of the asymmetries by varying the distributions within the bounds. By saturating the positivity bounds, one can obtain the upper and lower limits of the asymmetries.
- For the unpolarized distributions $f_{1}^{q}\left(x, k_{T}^{2}\right)$, we use the MSTW2008 LO set parametrization [75], and adopt a Gaussian form factor for the transverse momentum dependence which has been adopted in many phenomenological analyses [46, 47]

$$
f_{1}^{q}\left(x, k_{T}^{2}\right)=f_{1}^{q}(x) \frac{\exp \left(-k_{T}^{2} / k_{u n}^{2}\right)}{\pi k_{u n}^{2}},
$$

with $k_{u n}^{2}=0.25 \mathrm{GeV}^{2}$; the subscript "un" stands for the unpolarized distributions.

- In order to precisely predict the azimuthal asymmetries at different experiments using TMD factorization, it is essential for one to know the evolution of 3dPDFs. Unlike the PDFs in the collinear factorization approach, whose evolution has been well established by the DGLAP equation, the $Q^{2}$ dependence of $3 \mathrm{dPDF}$ is not fully understood yet. In our practical calculations we assume that the scale dependences of $3 \mathrm{dPDF}$ and the spin averaged distribution function $f_{1}$ are the same. The same assumption has been applied in some extractions of $3 \mathrm{dPDF}$ [33, 47-49]. To what extent that this approximation is valid still needs to be studied. As the asymmetries we calculate are ratios, we expect that our assumption on the scale dependence are reasonable.

Now we have all the ingredients for estimating the azimuthal asymmetries in single polarized proton-proton Drell-Yan processes. In the following, we apply the above ansatz to present our predictions and phenomenological 

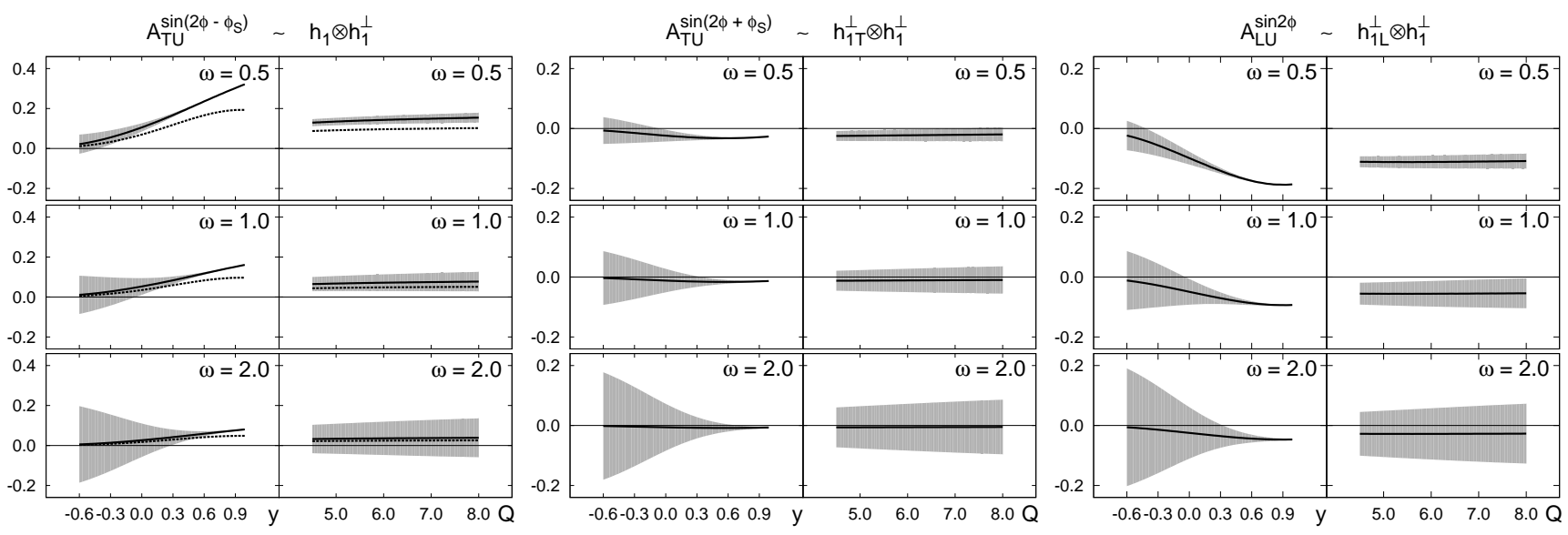

FIG. 4: Azimuthal asymmetries $A_{T U}^{\sin \left(2 \phi-\phi_{S}\right)}$ (left panels), $A_{T U}^{\sin \left(2 \phi+\phi_{S}\right)}$ (central panels), and $A_{L U}^{\sin 2 \phi}$ (right panels) at RHIC fixed-target experiments.

analysis for forthcoming experiments at RHIC, J-PARC, E906, and NICA.

\section{- RHIC}

The original proposal of Drell-Yan experiment at RHIC employs two proton beams to collide at $\sqrt{s}=200 \mathrm{GeV}$ or $500 \mathrm{GeV}$ 76]. But recently there is also a new proposal to conduct a fixed-target experiment at $\sqrt{s}=22 \mathrm{GeV}$ [77]. We estimate the asymmetries for both the collider and fixedtarget modes at RHIC. The longitudinal proton beam will be run in the coming years at RHIC, after that, Drell-Yan program with tranverse spin will be conducted. We choose the following kinematics for collider experiment at RHIC-STAR (Solenoidal Tracker at RHIC):

$$
\begin{aligned}
& \sqrt{s}=200 \mathrm{GeV}, \quad 4 \mathrm{GeV}<Q<9 \mathrm{GeV}, \\
& 0<q_{T}<1 \mathrm{GeV}, \quad-1<y<2 .
\end{aligned}
$$

We constrain the kinematical cut at the low transverse momentum region such that $q_{T}^{2} \ll Q^{2}$ where TMD factorization dominates.

In the left panels of Fig. 3 we show the estimated azimuthal asymmetries $A_{T U}^{\sin \left(2 \phi-\phi_{S}\right)}$ as functions of the rapidity $y$ and the dilepton mass $Q$, respectively. The difference between two linestyles in the left panels is that for the dashed lines we use the transversity distribution for valence $u$ and $d$ quarks from the parameterization in Ref. [33], while for the solid lines we adopt the results from the light-cone quark-diquark model [69, 70] for the valence distributions $h_{1}$. The thick solid and dashed lines correspond to the contribution merely from the combinations of the valence transversity distributions and the sea Boer-Mulders distributions, that is, ignoring the transversity distributions of sea quarks. The shaded regions give the ranges of $A_{T U}^{\sin \left(2 \phi-\phi_{S}\right)}$ by considering the additional contribution of the transversity distributions of sea quarks constrained by positivity bound (27). The upper and lower limits of the bands correspond to the asymmetries by saturating the positivity bound. The first, second, and third rows show the results for $\omega=0.5,1$ and 2, respectively, where $\omega$ is the parameter for Boer-Mulders functions, as explained previously. In the central and right panels of Fig. 3 we show the estimated azimuthal asymmetries $A_{T U}^{\sin \left(2 \phi+\phi_{S}\right)}$ and $A_{L U}^{\sin 2 \phi}$, respectively, in the same way as in the left panels.

From Fig. 3 we observe that in the forward rapidity region, the asymmetry $A_{T U}^{\sin \left(2 \phi-\phi_{S}\right)}$ at RHIC collider experiments is positive, while the asymmetries $A_{T U}^{\sin \left(2 \phi+\phi_{S}\right)}$ and $A_{L U}^{\sin 2 \phi}$ tend to be negative. It is interesting that the magnitudes of the asymmetries increase as the rapidity increases. At large forward rapidity, the asymmetry $A_{T U}^{\sin \left(2 \phi-\phi_{S}\right)}$ is dominated by the combination of the transversity of valence quarks and Boer-Mulders function of sea quarks (showed by the thick solid lines). This is understandable since large $y$ corresponds to larger $x_{1}$ and smaller $x_{2}$. Therefore the measurement of the asymmetries at large rapidity can provide the information of $T$-even chiral-odd distributions in valence region. Of course, the statistics at large rapidity are much lower than at midrapidity, therefore a reliable measurement requires data with high integrated luminosity. A common feature shared by all these asymmetries is that as $\omega$ increases, the asymmetry in the forward rapidity region tends to decrease. This arises from the fact that larger $\omega$ corresponds to larger valence Boer-Mulders function and smaller sea Boer-Mulders function.

In the left panels of Fig. 3, the magnitudes of the asymmetries $A_{T U}^{\sin \left(2 \phi-\phi_{S}\right)}$ calculated by using two 

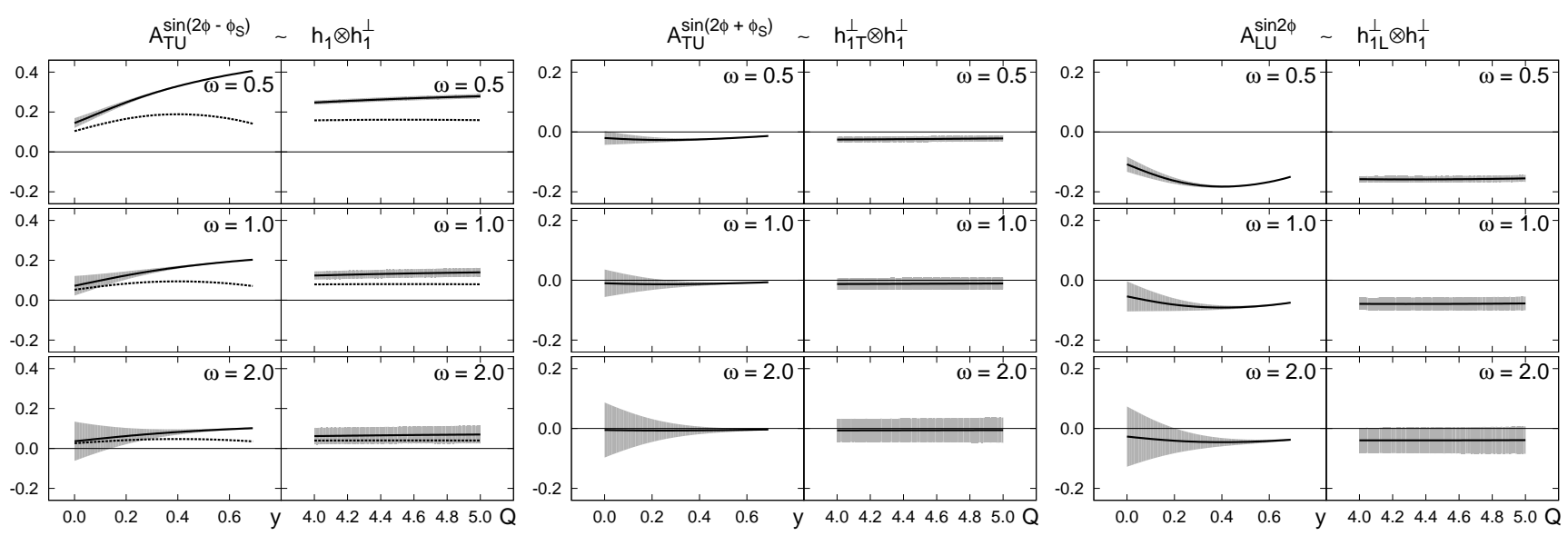

FIG. 5: Azimuthal asymmetries $A_{T U}^{\sin \left(2 \phi-\phi_{S}\right)}$ (left panels), $A_{T U}^{\sin \left(2 \phi+\phi_{S}\right)}$ (central panels), and $A_{L U}^{\sin 2 \phi}$ (right panels) at J-PARC.

different forms of the valence transversity distributions $h_{1}$ are quite different at large rapidity region. This is due to the fact that at large $x$ region the valence transversity distributions $h_{1}$ fitted by Anselmino et al. in Ref. 47] are smaller than the corresponding ones in the light-cone quark-diquark model [69, 70].

There is also the possibility of accelerating the polarized proton beam with $E_{p}=250 \mathrm{GeV}$ to collide on the proton target at RHIC. The RHIC kinematics for the fixed-target experiment are

$$
\begin{aligned}
& \sqrt{s}=22 \mathrm{GeV}, \quad 4.5 \mathrm{GeV}<Q<8 \mathrm{GeV}, \\
& 0<q_{T}<1 \mathrm{GeV}, \quad 0.2<x_{1}<0.6,
\end{aligned}
$$

corresponding to $-0.6<y<1.0$, which is complementary to the collider kinematics. In Fig. 4 we show the azimuthal asymmetries $A_{T U}^{\sin \left(2 \phi-\phi_{S}\right)}$ (left panels), $A_{T U}^{\sin \left(2 \phi+\phi_{S}\right)}$ (central panels), and $A_{L U}^{\sin 2 \phi}$ (right panels) at RHIC fixed-target Drell-Yan processes. It seems that the magnitude of the asymmetries $A_{T U}^{\sin \left(2 \phi-\phi_{S}\right)}$ and $A_{L U}^{\sin 2 \phi}$ at fixed-target experiments are larger than that at collider experiments. Therefore, there is a good chance to measure larger asymmetries at the fixed-target mode. The drawback is that, at fixed-target experiments, the uncertainty from the $T$-even chiral-odd distributions $h_{1}$, $h_{1 T}^{\perp}$, and $h_{1 L}^{\perp}$ of sea quarks at the negative rapidity region is larger than that at collider experiments. In both modes the asymmetries are consistent to zero at the large backward region and their size increases with the increase of the rapidity.

\section{- J-PARC}

J-PARC might measure azimuthal asymmetries given in Eqs. (18/19) in single polarized Drell-Yan processes at $E_{p}=50 \mathrm{GeV}$ 78], corresponding to $\sqrt{s} \simeq 10 \mathrm{GeV}$. The kinematical cuts at J-PARC are

$$
\begin{aligned}
& 4 \mathrm{GeV}<Q<5 \mathrm{GeV}, 0<q_{T}<1 \mathrm{GeV} \\
& 0.5<x_{1}<0.9
\end{aligned}
$$

corresponding to $0<y<0.69$. The estimated asymmetries $A_{T U}^{\sin \left(2 \phi-\phi_{S}\right)}, A_{T U}^{\sin \left(2 \phi+\phi_{S}\right)}$, and $A_{L U}^{\sin 2 \phi}$ are shown in the left, central, and right panels of Fig. 5. The figure manifests that the asymmetry $A_{T U}^{\sin \left(2 \phi-\phi_{S}\right)}$ is positive, while the asymmetry $A_{L U}^{\sin 2 \phi}$ is negative in all the allowed rapidity region. This feature can be seen even by considering the uncertainty at the midrapidity region from the distributions $h_{1}$ and $h_{1 L}^{\perp}$ of sea quarks. Larger asymmetries $A_{T U}^{\sin \left(2 \phi-\phi_{S}\right)}$ and $A_{L U}^{\sin 2 \phi}$ are predicted at J-PARC than those at RHIC. The asymmetry $A_{T U}^{\sin \left(2 \phi+\phi_{S}\right)}$ is much smaller than other two asymmetries, as at RHIC.

\section{- E906}

There is a new proposal to use proton beams from the main injector at $E_{p}=120 \mathrm{GeV}$ to collide on the polarized proton target $\left(\mathrm{NH}_{3}\right)$ by E906 Collaboration 79] at Fermi Lab. The polarized dimuon DrellYan program at E906 might be applied to measure the asymmetries defined in Eqs. (13), (14) and (15). The E906 kinematics are given as

$\sqrt{s}=15 \mathrm{GeV}, \quad 0.3<x_{1}<0.7, \quad 0.1<x_{2}<0.3$, $0<q_{T}<1 \mathrm{GeV}, 4 \mathrm{GeV}<Q<7 \mathrm{GeV}$,

corresponding to $0<y<0.76$. The calculated asymmetries $A_{T U}^{\sin \left(2 \phi-\phi_{S}\right)}, A_{T U}^{\sin \left(2 \phi+\phi_{S}\right)}$, and $A_{L U}^{\sin 2 \phi}$ are shown in the left, central, and right panels of Fig. 6 .

\section{- NICA}

NICA at JINR might realize both longitudinally and transversally polarized beams of protons at 

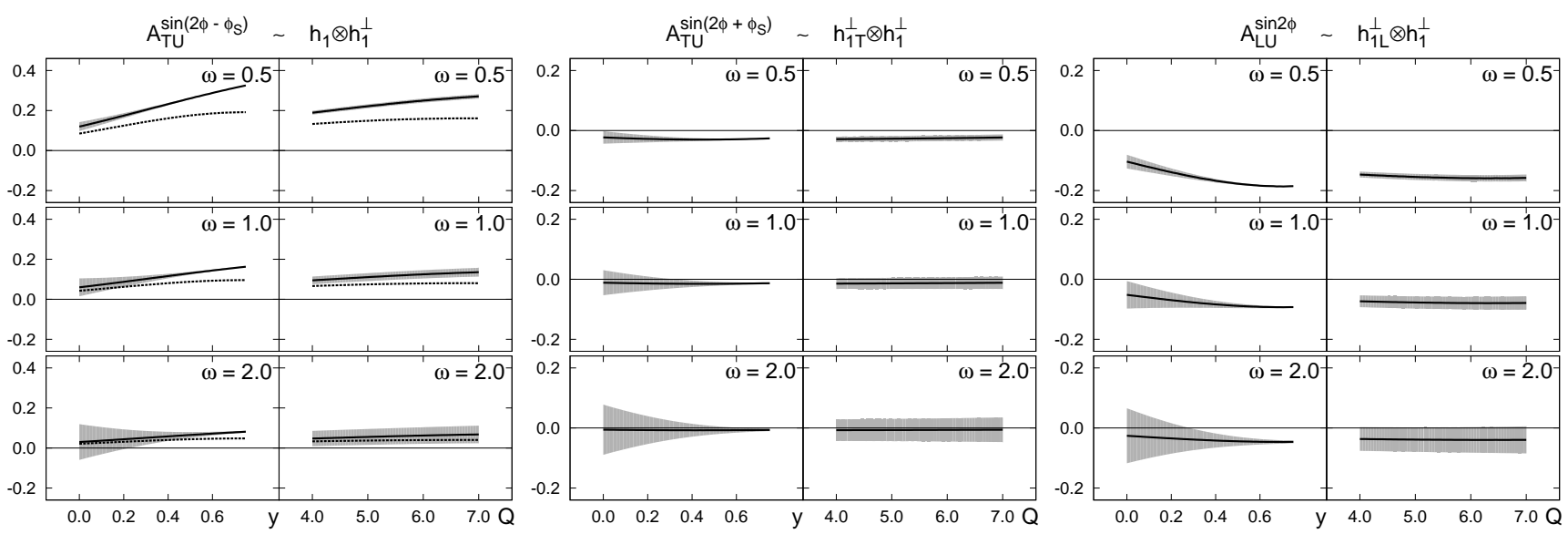

FIG. 6: Azimuthal asymmetries $A_{T U}^{\sin \left(2 \phi-\phi_{S}\right)}$ (left panels), $A_{T U}^{\sin \left(2 \phi+\phi_{S}\right)}$ (central panels), and $A_{L U}^{\sin 2 \phi}$ (right panels) at E906.

$\sqrt{s}=12 \sim 27 \mathrm{GeV}[80]$. The kinematics applied in our calculation at NICA are

$$
\begin{aligned}
& \sqrt{s}=27 \mathrm{GeV}, \quad 4 \mathrm{GeV}<Q<9 \mathrm{GeV}, \\
& 0<q_{T}<1 \mathrm{GeV}, \quad 0.1<x_{1}<0.8
\end{aligned}
$$

corresponding to $-1.1<y<1.1$. Here we choose the highest c.m. energy to avoid the overlap with other experiments. We present the asymmetries $A_{T U}^{\sin \left(2 \phi-\phi_{S}\right)}, A_{T U}^{\sin \left(2 \phi+\phi_{S}\right)}$, and $A_{L U}^{\sin 2 \phi}$ in the left, central, and left panels of Fig. 7, respectively.

Our predictions at RHIC, J-PARC, E906, and NICA show that the asymmetries $A_{T U}^{\sin \left(2 \phi-\phi_{S}\right)}, A_{T U}^{\sin \left(2 \phi+\phi_{S}\right)}$, and $A_{L U}^{\sin 2 \phi}$ are sensitive to the Boer-Mulders functions of sea quarks. This can be seen by comparing the plots in the three rows of each figure. The size of sea content can be described by the parameter $\omega$ appearing in the parametrizations of the Boer-Mulders functions. The case $\omega=1$ corresponds to the central values of the Boer-Mulders functions (which we refer as normal case), while $\omega=0.5$ corresponds to much smaller valence and much larger sea values (large sea case), and $\omega=2$ corresponds to much larger valence and much smaller sea values (small sea case) compared to the central values. In the normal case, we can see from the $Q$-dependent plots that the asymmetries $A_{T U}^{\sin \left(2 \phi-\phi_{S}\right)}$ and $A_{L U}^{\sin 2 \phi}$ are sizable at all entire allowed $Q$ regions. As $\omega$ increases or decreases, the asymmetries decrease or increase correspondingly. Therefore, their measurements could be used to discriminate different scenarios of the Boer-Mulders functions.

At larger backward rapidity region (RHIC and NICA) or midrapidity region (J-PARC and E906), the figures show that there are uncertainties contributed by the unknown sea content of $h_{1}, h_{1 T}^{\perp}$, and $h_{1 L}^{\perp}$ allowed by the positivity bounds, especially in the small sea case. In some cases the uncertainties are so large that the sizes and signs of the asymmetries can not been determined.
Precision measurement at these regions will provide further constraints on the sea content of $h_{1}, h_{1 T}^{\perp}$, and $h_{1 L}^{\perp}$.

The plots for $A_{T U}^{\sin \left(2 \phi-\phi_{S}\right)}$ and $A_{L U}^{\sin 2 \phi}$ show that these asymmetries are larger at the forward rapidity region, about $10 \%$ in magnitude in the normal case. Furthermore, our plots show that at the forward rapidity region, the contributions from the $T$-even chiral-odd distributions of valence quarks dominate, that is, they are less contaminated by their sea content. Hence the asymmetries at forward rapidity are measurable and the measurements on them are ideal to access the valence content of $h_{1}$, and $h_{1 L}^{\perp}$ at large $x$ region.

The magnitudes of the asymmetries $A_{T U}^{\sin \left(2 \phi-\phi_{S}\right)}$ are larger than $A_{L U}^{\sin 2 \phi}$ and $A_{T U}^{\sin \left(2 \phi+\phi_{S}\right)}$. This is because that the size of the transversity distributions in the light-cone quark-diquark model is larger than that of $h_{1 T}^{\perp}$ and $h_{1 L}^{\perp}$. The comparison of different types of asymmetries might be used to distinguish the sizes of different $T$-even chiralodd distributions and to check the approximate relations among TMDs [81].

\section{CONCLUSION}

We have studied the azimuthal asymmetries in the single polarized proton-proton Drell-Yan processes by considering particularly the contributions of the leadingtwist chiral-odd quark distributions, i.e., the BoerMulders function, transversity, pretzlosity and longitudinal transversity. We define the azimuthal asymmetries $A_{T U}^{\sin \left(2 \phi+\phi_{S}\right)}$ and $A_{T U}^{\sin \left(2 \phi-\phi_{S}\right)}$ in transverse single polarized $p^{\uparrow} p$ Drell-Yan processes, and $A_{L U}^{\sin 2 \phi}$ asymmetry in the longitudinal single polarized $p \rightarrow p$ Drell-Yan processes. Using the predictions for the transversity, pretzlosity and longitudinal transversity from the light-cone quark-diquark model, and the Boer-Mulders functions extracted from the unpolarized Drell-Yan data at low transverse momentum, we present a comprehensive phe- 

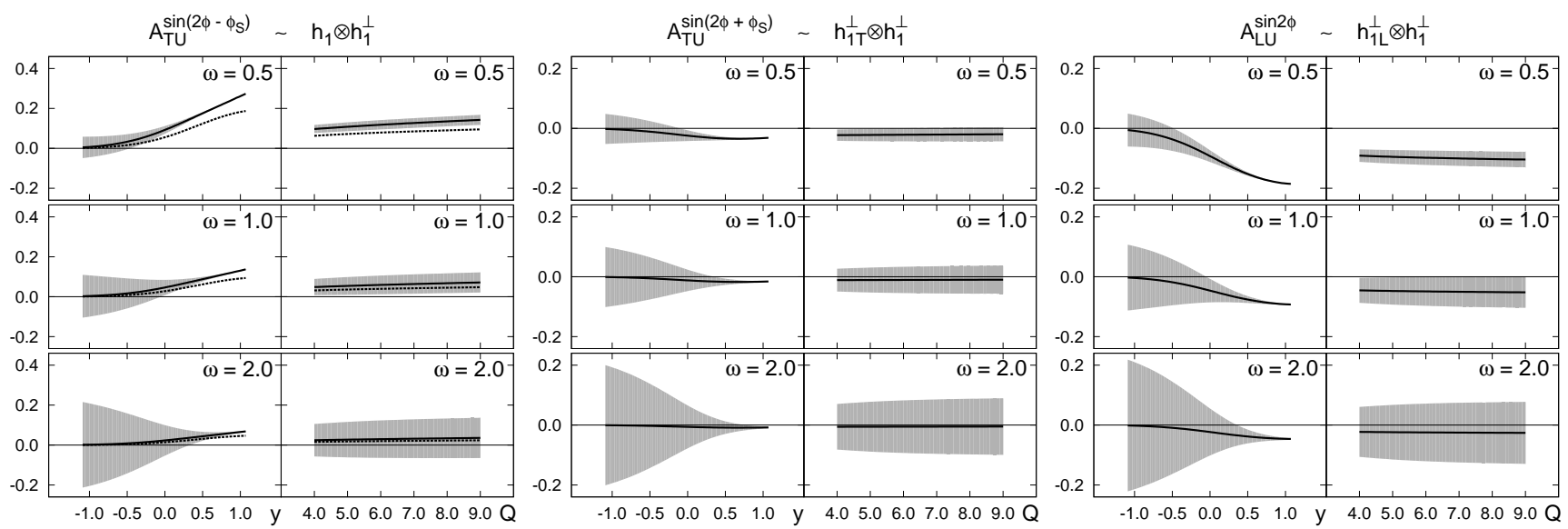

FIG. 7: Azimuthal asymmetries $A_{T U}^{\sin \left(2 \phi-\phi_{S}\right)}$ (left panels), $A_{T U}^{\sin \left(2 \phi+\phi_{S}\right)}$ (central panels), and $A_{L U}^{\sin 2 \phi}$ (right panels) at NICA.

nomenological analysis of the asymmetries $A_{T U}^{\sin \left(2 \phi+\phi_{S}\right)}$, $A_{T U}^{\sin \left(2 \phi-\phi_{S}\right)}$, and $A_{L U}^{\sin 2 \phi}$ at RHIC, J-PARC, E906, and NICA. In all these facilities there are polarized Drell-Yan programs in preparation or being planned, including collider experiments (RHIC and NICA) and fixed-target experiments (RHIC, J-PARC, and E906). Our study shows that the polarized Drell-Yan programs at various facilities can be used to explore the valence and sea content of the leading-twist chiral-odd distributions in wide kinematical regions.

\section{Acknowledgement}

This work is partially supported by National Natural Science Foundation of China (Grants No. 10905059, No. 11005018, No. 11021092, No. 10975003, No. 11035003, and No. 11120101004) and by FONDECYT (Chile) under Project No. 11090085.
[1] V. Barone, A. Drago, and P. G. Ratcliffe, Phys. Rep. 359, 1 (2002).

[2] U. D'Alesio and F. Murgia, Prog. Part. Nucl. Phys. 61, 394 (2008).

[3] V. Barone, F. Bradamante, and A. Martin, Prog. Part. Nucl. Phys. 65, 267 (2010).

[4] D. Boer, M. Diehl, R. Milner, R. Venugopalan, W. Vogelsang, A. Accardi, E. Aschenauer, and M. Burkardt et al., arXiv:1108.1713 [nucl-th].

[5] D. L. Adams et al., (FNAL E581 and E704 Collaborations), Phys. Lett. B 261, 201 (1991); Phys. Lett. B 276, 531 (1992); Z. Phys. C 56, 181 (1992); D. L. Adams et al. (FNAL E704 Collaboration), Phys. Lett. B 264, 462 (1991); Phys. Rev. D 53, 4747 (1996); A. Bravar et al. (FNAL E704 Collaboration), Phys. Rev. Lett. 77, 2626 (1996).

[6] G. L. Kane, J. Pumplin, and W. Repko, Phys. Rev. Lett. 41, 1689 (1978).

[7] C. Boros, Z. Liang, and T. Meng, Phys. Rev. Lett. 70, 1751 (1993).

[8] D. Sivers, Phys. Rev. D 41, 83 (1990); 43, 261(E) (1991).

[9] M. Anselmino, M. Boglione, and F. Murgia, Phys. Lett. B 362, 164 (1995); M. Anselmino and F. Murgia, Phys. Lett. B 442, 470 (1998).

[10] The Trento conventions have been used, see A. Bacchetta, U. D'Alesio, M. Diehl, and C. A. Miller, Phys. Rev. D 70, 117504 (2004).
[11] J. C. Collins, Nucl. Phys. B396, 161 (1993).

[12] S. J. Brodsky, D. S. Hwang, and I. Schmidt, Phys. Lett. B 530, 99 (2002); Nucl. Phys. B642, 344 (2002).

[13] X. Ji and F. Yuan, Phys. Lett. B 543, 66 (2002); A. V. Belitsky, X. Ji, and F. Yuan, Nucl. Phys. B656, 165 (2003).

[14] D. Boer, P. J. Mulders, and F. Pijlman, Nucl. Phys. B667, 201 (2003).

[15] J. C. Collins, Phys. Lett. B 536, 43 (2002).

[16] J. Collins and J. -W. Qiu, Phys. Rev. D 75, 114014 (2007).

[17] J. Collins, arXiv:0708.4410 [hep-ph].

[18] W. Vogelsang and F. Yuan, Phys. Rev. D 76, 094013 (2007).

[19] C. J. Bomhof and P. J. Mulders, Nucl. Phys. B795, 409 (2008).

[20] T. C. Rogers, P. J. Mulders, Phys. Rev. D 81, 094006 (2010).

[21] A. Bravar (Spin Muon Collaboration), Nucl. Phys. A666, 314 (2000).

[22] A. Airapetian et al., (HERMES Collaboration), Phys. Rev. Lett. 94, 012002 (2005).

[23] V. Y. Alexakhin et al., (COMPASS Collaboration), Phys. Rev. Lett. 94, 202002 (2005).

[24] A. Airapetian et al. (HERMES Collaboration), Phys. Lett. B 693, 11 (2010).

[25] E. S. Ageev et al., (COMPASS Collaboration), Nucl. 
Phys. B765, 31 (2007).

[26] A. Airapetian et al., (HERMES Collaboration), Phys. Rev. Lett. 103, 152002 (2009).

[27] M. G. Alekseev et al., (COMPASS Collaboration), Phys. Lett. B 692, 240 (2010).

[28] X. Qian et al., (The Jefferson Lab Hall A Collaboration), Phys. Rev. Lett. 107, 072003 (2011).

[29] M. Anselmino, M. Boglione, U. D’Alesio, A. Kotzinian, F. Murgia, and A. Prokudin, Phys. Rev. D 72, 094007 (2005); 72, 099903(E) (2005).

[30] A. V. Efremov, K. Goeke, S. Menzel, A. Metz, and P. Schweitzer, Phys. Lett. B 612, 233 (2005).

[31] J. C. Collins, A. V. Efremov, K. Goeke, S. Menzel, A. Metz, and P. Schweitzer, Phys. Rev. D 73, 014021 (2006).

[32] W. Vogelsang and F. Yuan, Phys. Rev. D 72, 054028 (2005).

[33] M. Anselmino, M. Boglione, U. D'Alesio, A. Kotzinian, S. Melis, F. Murgia, A. Prokudin, and C. Turk, Eur. Phys. J. A 39, 89 (2009).

[34] X. Ji, J. P. Ma, and F. Yuan, Phys. Rev. D 71, 034005 (2005); Phys. Lett. B 597, 299 (2004).

[35] J. C. Collins and A. Metz, Phys. Rev. Lett. 93, 252001 (2004).

[36] M. Anselmino, M. Boglione, U. D'Alesio, S. Melis, F. Murgia, and A. Prokudin, Phys. Rev. D 79, 054010 (2009).

[37] Z. -B. Kang, J. -W. Qiu, W. Vogelsang, F. Yuan, Phys. Rev. D83, 094001 (2011).

[38] S. Arnold, A. Metz, and M. Schlegel, Phys. Rev. D 79, 034005 (2009).

[39] D. Boer, Phys. Rev. D 60, 014012 (1999).

[40] J. P. Ralston and D. E. Soper, Nucl. Phys. B152, 109 (1979).

[41] R. L. Jaffe and X. -D. Ji, Phys. Rev. Lett. 67, 552 (1991).

[42] D. Boer and P. J. Mulders, Phys. Rev. D 57, 5780 (1998).

[43] P. J. Mulders and R. D. Tangerman, Nucl. Phys. B461, 197 (1996); B484, 538(E) (1997).

[44] A. Bacchetta, M. Diehl, K. Goeke, A. Metz, P. J. Mulders, and M. Schlegel, J. High Energy Phys 02, 093 (2007).

[45] K. Goeke, A. Metz, and M. Schlegel, Phys. Lett. B 618, 90 (2005).

[46] M. Anselmino, M. Boglione, U. D'Alesio, A. Kotzinian, F. Murgia, A. Prokudin, and C. Turk, Phys. Rev. D 75, 054032 (2007).

[47] M. Anselmino, M. Boglione, U. D'Alesio, A. Kotzinian, F. Murgia, A. Prokudin, and S. Melis, Nucl. Phys. Proc. Suppl. 191, 98 (2009).

[48] B. Zhang, Z. Lu, B. -Q. Ma, and I. Schmidt, Phys. Rev. D 77, 054011 (2008).

[49] Z. Lu and I. Schmidt, Phys. Rev. D 81, 034023 (2010).

[50] V. Barone, S. Melis, and A. Prokudin, Phys. Rev. D 81, 114026 (2010).

[51] V. Barone, S. Melis, and A. Prokudin, Phys. Rev. D 82, 114025 (2010).
[52] B. Pasquini, S. Cazzaniga, and S. Boffi, Phys. Rev. D 78, 034025 (2008).

[53] A. Bacchetta, F. Conti, and M. Radici, Phys. Rev. D 78, 074010 (2008).

[54] H. Avakian, A. V. Efremov, P. Schweitzer, and F. Yuan, Phys. Rev. D 78, 114024 (2008).

[55] J. She, J. Zhu, and B. -Q. Ma, Phys. Rev. D 79, 054008 (2009).

[56] A. V. Efremov, P. Schweitzer, O. V. Teryaev, and P. Zavada, Phys. Rev. D 80, 014021 (2009).

[57] S. Boffi, A. V. Efremov, B. Pasquini, and P. Schweitzer, Phys. Rev. D 79, 094012 (2009).

[58] H. Avakian, A. V. Efremov, P. Schweitzer, and F. Yuan, Phys. Rev. D 81, 074035 (2010).

[59] J. Zhu and B. -Q. Ma, Phys. Lett. B 696, 246 (2011).

[60] J. Zhu and B. -Q. Ma, Phys. Rev. D 82, 114022 (2010).

[61] J. C. Collins and D. E. Soper, Nucl. Phys. B193, 381 (1981); B213, 545(E) (1983).

[62] J. C. Collins and D. E. Soper, Phys. Rev. D 16, 2219 (1977).

[63] A. Bacchetta, M. Radici, F. Conti, and M. Guagnelli, Eur. Phys. J. A 45, 373 (2010).

[64] L. Y. Zhu, et. al., (FNAL-E866/NuSea Collaboration), Phys. Rev. Lett. 99, 082301 (2007).

[65] L. Y. Zhu et al. (FNAL E866/NuSea Collaboration), Phys. Rev. Lett. 102, 182001 (2009).

[66] E. P. Wigner, Annals Math. 40, 149 (1939); H. J. Melosh, Phys. Rev. D 9, 1095 (1974).

[67] B. -Q. Ma, J. Phys. G 17, L53 (1991), arXiv:0711.2335 [hep-ph]]; B. -Q. Ma and Q. -R. Zhang, Z. Phys. C 58, 479 (1993).

[68] B. -Q. Ma, Phys. Lett. B 375, 320 (1996).

[69] B. -Q. Ma, I. Schmidt, and J. Soffer, Phys. Lett. B 441, 461 (1998).

[70] I. Schmidt and J. Soffer, Phys. Lett. B 407, 331 (1997).

[71] Z. Lu and B. -Q. Ma, Nucl. Phys. A741, 200 (2004).

[72] B. -Q. Ma, I. Schmidt, and J. -J. Yang, Phys. Rev. D 65, 034010 (2002).

[73] B. -Q. Ma, I. Schmidt, and J. -J. Yang, Phys. Rev. D 66, 094001 (2002).

[74] A. Bacchetta, M. Boglione, A. Henneman, and P. J. Mulders, Phys. Rev. Lett. 85, 712 (2000).

[75] A. D. Martin, W. J. Stirling, R. S. Thorne, and G. Watt, Eur. Phys. J. C 63, 189 (2009).

[76] G. Bunce, N. Saito, J. Soffer, and W. Vogelsang, Ann. Rev. Nucl. Part. Sci. 50, 525 (2000).

[77] Y. Goto, J. Phys. Conf. Ser. 295, 012166 (2011).

[78] Y. Goto, Proc. Sci., DIS2010, 264 (2010).

[79] M. X. Liu, X. Jiang, D. G. Crabb, J. P. Chen, and M. Bai, J. Phys. Conf. Ser. 295, 012164 (2011).

[80] I. N. Meshkov and Yu. N. Filatov, J. Phys. Conf. Ser. 295, 012144 (2011).

[81] H. Avakian, A. V. Efremov, K. Goeke, A. Metz, P. Schweitzer, and T. Teckentrup, Phys. Rev. D 77, 014023 (2008). 